\title{
Simultaneous enhancement of chemical shift dispersion and diffusion resolution in mixture analysis by diffusion-ordered NMR spectroscopy
}

\author{
Alexandria K. Rogerson, Juan A. Aguilar, Mathias Nilsson and Gareth A. Morris* \\ Received 27th April 2011, Accepted 18th May 2011 \\ DOI: $10.1039 / \mathrm{clcc12456k}$
}

\begin{abstract}
Mixture analysis by high resolution diffusion-ordered NMR spectroscopy (HR-DOSY) requires differences in both chemical shift and diffusion coefficient; resolution can be greatly enhanced by exploiting the chemical specificity of lanthanide shift reagent binding to increase chemical shift and diffusion dispersion simultaneously.
\end{abstract}

NMR spectroscopy is a powerful tool for the elucidation of molecular structure, but has been little used for mixture analysis owing to the difficulty of distinguishing resonances from individual species. Diffusion-ordered spectroscopy $(D O S Y)^{1-3}$ allows the separation of signals from different species where they have different diffusion coefficients $D$. In DOSY, a series of pulsed field gradient stimulated echo experiments is carried out, and the results are used to synthesise a two-dimensional spectrum where signals are dispersed according to their diffusion coefficients, ${ }^{4} \mathrm{D}$ for each signal is typically found by fitting the observed attenuation to the Stejskal-Tanner ${ }^{5}$ equation

$$
S=S_{0} e^{-D \gamma^{2} \delta^{2} G^{2} \Delta^{\prime}}
$$

where $S$ is the signal amplitude, $S_{0}$ is the amplitude that would have resulted without diffusion, $\delta$ is the gradient pulse width, $\gamma$ is the magnetogyric ratio, $G$ is the gradient amplitude, and $\Delta^{\prime}$ is the effective diffusion time.

High resolution in DOSY requires both that mixture components have different diffusion coefficients, and that their signals do not overlap. Diffusion coefficients of different mixture components can if necessary be manipulated by changing the matrix in which they diffuse (matrix-assisted $\operatorname{DOSY}^{6-8}$ ). Signal overlap is more problematic; various data processing methods, including multiexponential fitting ${ }^{9}$ and multivariate methods ${ }^{10-13}$ are available to mitigate its effects, but much the best solution is to avoid it. One powerful method for reducing overlap is to extend DOSY spectra into a third dimension, ${ }^{14-16}$ but this is very expensive in experiment time. In 2D DOSY, "pure shift" methods greatly improve resolution by suppressing homonuclear multiplet structure ${ }^{15,17}$ but only at a significant cost in signal-to-noise ratio. ${ }^{13} \mathrm{CDOSY}^{18}$ gives

School of Chemistry, University of Manchester, Oxford Road,

Manchester,M139PL,UK.E-mail:g.a.morris@manchester.ac.uk much better resolution than ${ }^{1} \mathrm{H}$, but again at a high cost in sensitivity.

Here a novel approach is proposed that simultaneously addresses both requirements. It is well known that lanthanide shift reagents (LSRs) can enhance chemical shift resolution by binding to Lewis basic sites in substrates, ${ }^{19-21}$ but this binding also modulates diffusion behaviour by forming a bulky complex. Thus the chemically-selective binding of LSRs to mixture components can simultaneously enhance chemical shift and diffusion resolution in DOSY. (Binding also affects relaxation, an effect that has been exploited using diamagnetic analogues of $\mathrm{LSRs}^{22}$ ).

In this communication we demonstrate DOSY resolution enhancement using a mixture of species with similar size and severe overlap in chemical shift. Fig. 1(a) and (b) show Oneshot $45^{23,24}$ DOSY spectra of a mixture of n-hexane, hexan-1-ol and n-heptanal, respectively with and without the LSR Eu(fod $)_{3}$. In Fig. 1(a) the area from 0 to 3 ppm shows severe overlap of resonances from all three species. As a result, most signals show similar apparent diffusion; here the components are effectively indistinguishable, overlap obscuring the fact that hexane diffuses slightly faster than the other two components.

In Fig. 1(b), the addition of $\mathrm{Eu}(\mathrm{fod})_{3}$ not only removes signal overlap, revealing the rapid diffusion (red line) of the hexane, which does not bind to the LSR, but also resolves the hexanol (green) and heptanal (blue) signals in the diffusion dimension because the former, as a stronger Lewis base, binds more tightly to the LSR (yellow) and hence diffuses more slowly. Here the choice of LSR concentration requires a compromise between increased chemical shift dispersion and increasing signal linewidth; higher and lower concentrations than that used for Fig. 1(b) gave mostly well-resolved DOSY spectra but with some residual signal overlap.

Measurements were carried out non-spinning on a Varian VNMRS $500 \mathrm{MHz}$ spectrometer at ambient temperature, using the Oneshot45 DOSY pulse sequence. ${ }^{23,24}$ Data were acquired in $35 \mathrm{~min}$ with 16 nominal gradient amplitudes ranging from 3.0 to $27.0 \mathrm{G} \mathrm{cm}^{-1}$ in equal steps of gradient squared, using 16 transients, 65536 complex data points, a total diffusion-encoding gradient duration $\delta$ of $1 \mathrm{~ms}$ and a diffusion time $\Delta$ of $0.2 \mathrm{~s}$. DOSY spectra were constructed using correction for the effects of pulsed field gradient non-uniformity. ${ }^{25}$ Reference deconvolution $^{26}$ was used for Fig. 1(a) with a 

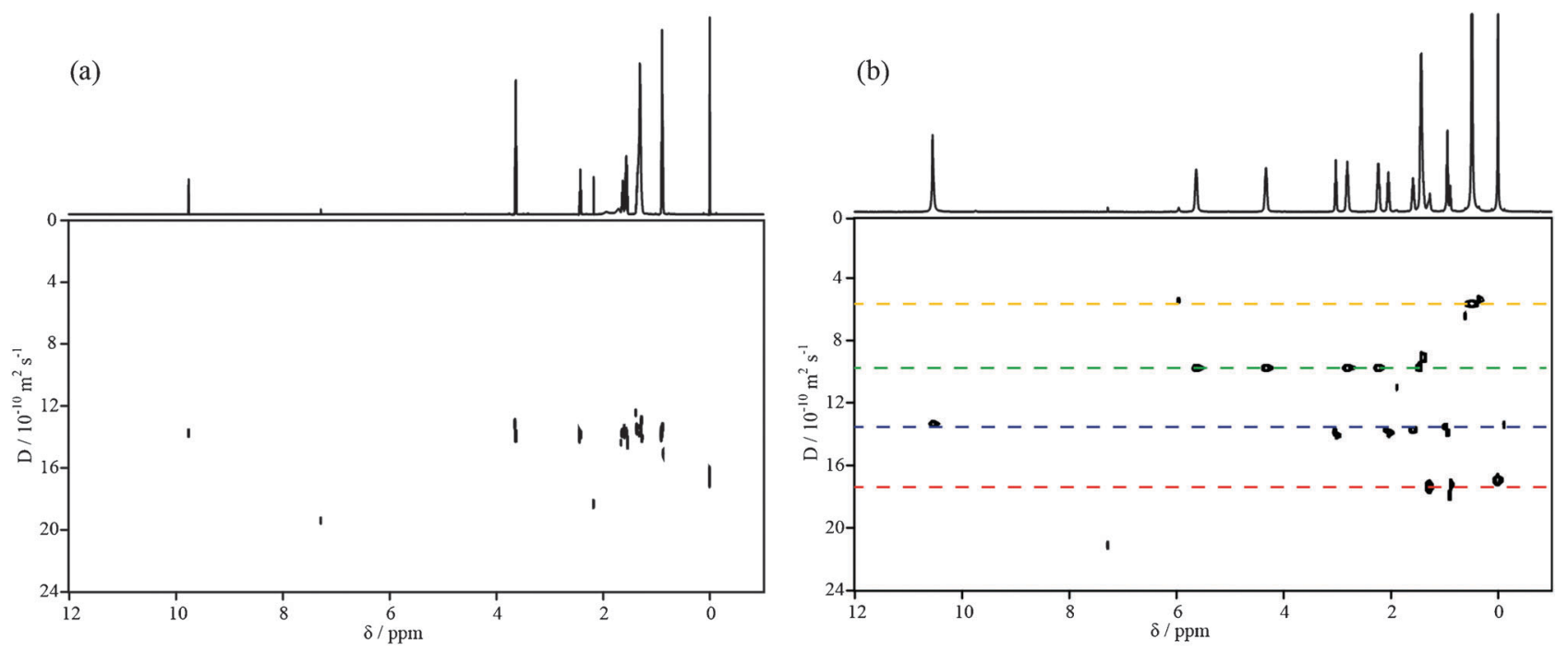

Fig. 1 Oneshot45 DOSY spectra of a mixture containing n-hexane (60 mM), hexan-1-ol (100 mM), and n-heptanal (100 mM) and TMS reference in $\mathrm{CDCl}_{3}$, without (a) and with (b) the addition of $50 \mathrm{mM} \mathrm{Eu}(\mathrm{fod})_{3}$. In Fig. 1(b), the diffusion coefficients of Eu(fod) $)_{3}$, hexanol, heptanal and hexane are indicated by yellow, green, blue and red dotted lines, respectively; assignments were confirmed using COSY.

Gaussian target lineshape of width $0.8 \mathrm{~Hz}$; overlap between the TMS and $\mathrm{Eu}(\mathrm{fod})_{3}$ signals prevented reference deconvolution of the data of Fig. 1(b), so Lorentz-Gauss transformation corresponding to $-1 \mathrm{~Hz}$ Lorentzian linewidth and $2 \mathrm{~Hz}$ Gaussian linewidth was used.

In chemically compatible systems, the use of LSRs in matrix-assisted DOSY can yield a great improvement in resolution. The method will work best where the LSR is significantly larger than the mixture components; it would be straightforward to extend the method to larger molecules by increasing the LSR size. The effects of LSRs on chemical shift and on diffusion are relatively well-understood, suggesting applications in the analysis of mixtures of unknowns; given the greater linewidths at high field, ${ }^{27}$ low and medium field studies should benefit most. There is also potential for extension into a third dimension, exploiting the relaxation effects of LSRs by analogy with $\mathrm{T}_{1}$-DOSY. ${ }^{28}$ Many other classes of co-solute could be exploited in a similar manner, for example cyclodextrins and diamagnetic porphyrins.

This work was supported by the Engineering and Physical Sciences Research Council (Grant Numbers EP/H024336/1, EP/I007989/1, and EP/E05899X/1). AKR thanks the EPSRC for a DTA studentship.

\section{Notes and references}

1 C. S. Johnson, Prog. Nucl. Magn. Reson. Spectrosc., 1999, 34, 203-256.

2 G. A. Morris, Diffusion-Ordered Spectroscopy (DOSY), in Encyclopedia of Magnetic Resonance, ed. R. K. Harris and R. E. Wasylishen, John Wiley \& Sons, Ltd., Chichester, UK, 2009, DOI: 10.1002/ 9780470034590.emrstm0119.pub2.

3 Y. Cohen, L. Avram and L. Frish, Angew. Chem., Int. Ed., 2005, 44, 520-554.

4 B. Antalek, Concepts Magn. Reson., 2002, 14, 225-258.

5 E. O. Stejskal and J. E. Tanner, J. Chem. Phys., 1965, 42, 288-292.
6 M. E. Zielinski and K. F. Morris, Magn. Reson. Chem., 2009, 47, 53-56.

7 R. Evans, S. Haiber, M. Nilsson and G. A. Morris, Anal. Chem., 2009, 81, 4548-4550.

8 C. F. Tormena, R. Evans, S. Haiber, M. Nilsson and G. A. Morris, Magn. Reson. Chem., 2010, 48, 550-553.

9 M. Nilsson, M. A. Connell, A. L. Davis and G. A. Morris, Anal. Chem., 2006, 78, 3040-3045.

10 M. Nilsson and G. A. Morris, Anal. Chem., 2008, 80, 3777-3782.

11 P. Stilbs and K. Paulsen, Rev. Sci. Instrum., 1996, 67, 4380-4386.

12 W. Windig, J. P. Hornak and B. Antalek, J. Magn. Reson., 1998, 132, 298-306.

13 M. Nilsson, J. Magn. Reson., 2009, 200, 296-302.

14 D. Wu, A. Chen and C. S. Johnson, J. Magn. Reson., Ser. A, 1996, 121, 88-91.

15 M. Nilsson, A. M. Gil, I. Delagadillo and G. A. Morris, Chem. Commun., 2005, 1737-1739.

16 S. Viel and S. Caldarelli, Chem. Commun., 2008, 2013-2015.

17 M. Nilsson and G. A. Morris, Chem. Commun., 2007, 933-935; J. A. Aguilar, S. Faulkner, M. Nilsson and G. A. Morris, Angew. Chem. Int. Ed., 2010, 49, 3901-3903.

18 J. A. Aguilar, S. Faulkner, M. Nilsson and G. A. Morris, J. Magn. Reson., DOI: 10.1016/j.jmr.2011.1003.1016, in press.

19 C. C. Hinckley, J. Am. Chem. Soc., 1969, 91, 5160-5162.

20 J. K. M. Sanders and D. H. Williams, Nature, 1972, 240, 385-390.

21 B. C. Mayo, Chem. Soc. Rev., 1973, 2, 49-74.

22 J. D. Mersh and J. K. M. Sanders, J. Magn. Reson., Ser A, 1983, 51, 345-347.

23 M. D. Pelta, G. A. Morris, M. J. Stchedroff and S. J. Hammond, Magn. Reson. Chem., 2002, 40, S147-S152.

24 A. Botana, J. A. Aguilar, M. Nilsson and G. A. Morris, J. Magn. Reson., 2011, 208, 270-278.

25 M. A. Connell, P. J. Bowyer, P. A. Bone, A. L. Davis, A. G. Swanson, M. Nilsson and G. A. Morris, J. Magn. Reson., 2009, 198, 121-131.

26 G. A. Morris, H. Barjat and T. J. Horne, Prog. Nucl. Magn. Reson. Spectrosc., 1997, 31, 197-257.

27 J. M. Bulsing, J. K. M. Sanders and L. D. Hall, J. Chem. Soc., Chem. Commun., 1981, 1201.

28 M. Nilsson, A. Botana and G. A. Morris, Anal. Chem., 2009, 81, $8119-8125$. 